\title{
CONTROL MOUSE AND COMPUTER SYSTEM USING VOICE COMMANDS
}

\author{
Mahammad Rafi ${ }^{1}$, Khan Sohail Ahmed ${ }^{2}$, Shaikh Huda ${ }^{3}$, Lutful Islam ${ }^{4}$ \\ ${ }^{1}$ Student,Computer, Mohammad Haji Saboo Siddik College of Engineering, Maharashtra, India \\ ${ }^{2}$ Student, Computer, Mohammad Haji Saboo Siddik College of Engineering, Maharashtra, India \\ ${ }^{3}$ Student, Computer, Mohammad Haji Saboo Siddik College of Engineering, Maharashtra, India \\ ${ }^{4}$ Assistant Professor, Computer, Mohammad Haji Saboo Siddik College of Engineering, Maharashtra, India
}

\begin{abstract}
In this paper we propose a new product "Control Mouse and Computer System Using Voice Commands". It can be used to operate the entire computer functions on the user's voice commands. It makes use of the Speech Recognition technology that allows the computer system to identify and recognize words spoken by a human using a microphone. This Software will be able to recognize your spoken words and enable user to interact with his computer. This interaction includes user giving commands to his computer which will then respond by performing several tasks, actions or operations depending on the commands you gave. It can also help user to dictate and computer would convert their spoken words into text for word processing application or E-mail. For Example: Opening /closing a file, starting an application, Mouse click or movement.
\end{abstract}

Keywords: Speech Technology, Voice Response System, Voice Commands, Human-Computer Verbal Interaction. $* * *$

\section{INTRODUCTION}

Speech Recognition is a technology which helps computer to understand the words spoken by its user. The aim of this technology is to be able to understand the words spoken by user and provide human-verbal interaction. [1]

Even after years of research in this area, softwares using speech recognition still cannot recognize user's speech with complete accuracy. There are few applications which can recognize over $80 \%$ of words and only when spoken under some specific conditions. Hence, there are only limited uses of this technology.

Our product uses this technology to provide a naïve user the ability to interact with computer. Since speech is man's most effective form of communication, then why not use it to communicate with the computer? Our Software makes that happen by allowing you to have conversations with the computer.

This conversation involves you giving some commands and the computer responding by some actions or operations based on the commands.

Our product includes the following characteristics:

- Multi user voice recognition

- Better accuracy as compared to other existing systems

- Works for game applications
- Comprises of an enhanced dictionary with more than 1500 words

- Comprises of 1000 plus commands

- Works for web applications-Facebook

This System also helps physically challenged users who cannot use the keyboard and mouse. For example: Users without hands, or without eyesight().Control Mouse and Computer System Using Voice Commandsallow dictation as well as control of many computer tasks.

\section{LITERATURE SURVEY}

Human-computer interfaces facilitate communication, assist on the exchange of information, process commands and controls and perform several additional interactions. Spoken natural language is more user-friendly mean of interacting with a computer. From the human perspective point, this kind of interaction is easier since it does not urge humans to learn additional interactions. Humans can rely on natural ways of communicationsinstead.

Human-computer interaction varies from understanding simple commands to extracting all the information in the speech signal such as words, meanings and emotions of the user. To develop an interface with natural language understanding ability, several factors arise and must be taken into accountsuch as dealing with the ungrammatical nature of many spoken utterances, the detection of problems in speech recognition, and the design of intelligent clarification dialogues. 
There are also verbal interaction problems such as background noise, word echoing and repetition, and different and background sound sources overlapping the speech. Solution for these problems must be developed. [2]

Most state-of-the-art of the human-computer verbal interaction based systems relies on using simple voice commands, hence may not be considered as complete speech systems. Having these concepts in mind and addressing human-computer verbal issues, the speech recognition products should be well developed and be resilient enough for effective human-computer verbal interaction.

The system we are proposing here consists of providing an augmented speech dialogue structure used as and tested to study verbal human-computer interaction (user inputs computer responds to the command by performing the desired task).

\section{PROPOSED SYSTEM}

The principle aim of our project is to create a userindependent automatic speech recognizer with an altered grammar unit.

The system will be able to retrieve folders, sub-folders, documents \& other application/software using voice commands. Our system can also launch any file or macro. Dictation of texts using various products like Microsoft Office, Notepad \& other Text editors is also made possible through our system.

Our software helps open websites, documents, or programs. Simulation of keystrokes and running of any file is also made possible. By voice commands we can enable shutdown, restart or log off operations on the computer. [3]

It is a thrilling technology that will change the way computer user's interaction with the computer. User can speak to his computer and it will respond by performing the tasks ordered by the user. The speech that user and his computer interact with is scripted. In other words, user can talk to his computer using a set of predefined commands and instructions given in the grammar (i.e., a script). For example, you can say: "Mouse Left" and the computer would respond by moving the mouse cursor left. Or you can say: "My Computer" and the computer would open the "My Computer". As for the programming language we used Microsoft's Visual Basic.NET. Also we are going to make use of Microsoft Speech Application Programming Interface-SDK. We will try to take minimum required inputs from the user \& will give the desired output in the system.

\section{IMPLEMENTATION}

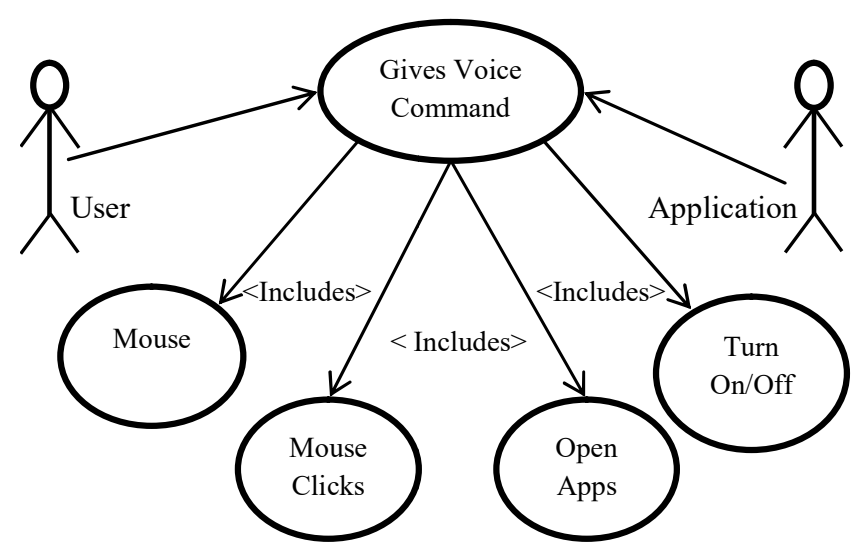

1: Use Case Diagram

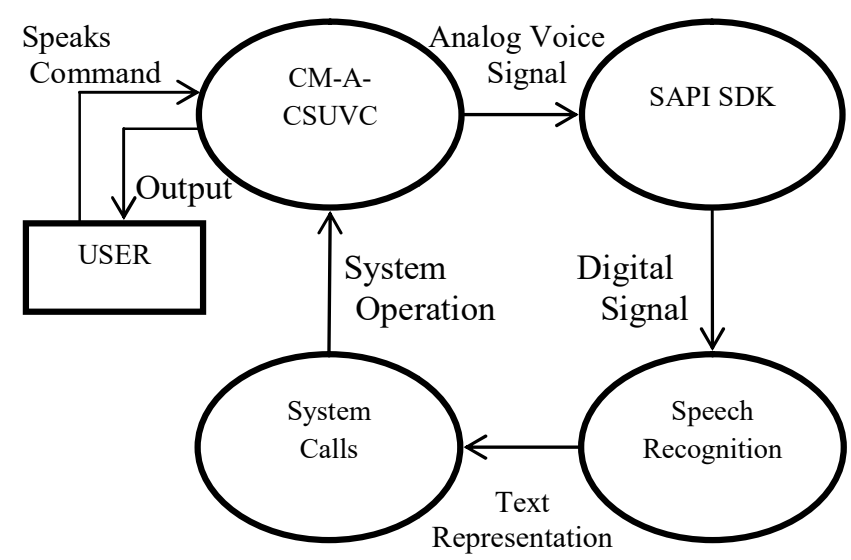

2: DFD Level 1

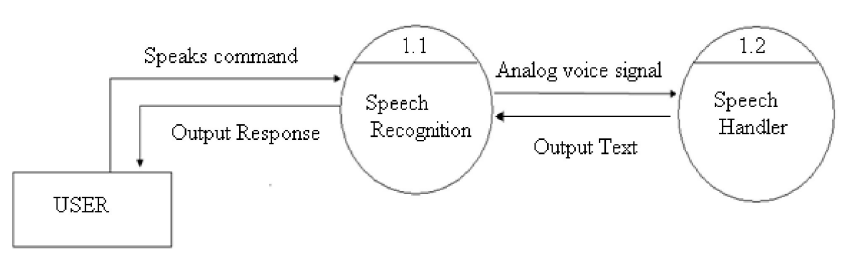

FIG: Level 2 Data Flow Diagram

3: DFD Level 2 


\section{FLOWCHART AND ALGORITHM}

\subsection{Flowchart}

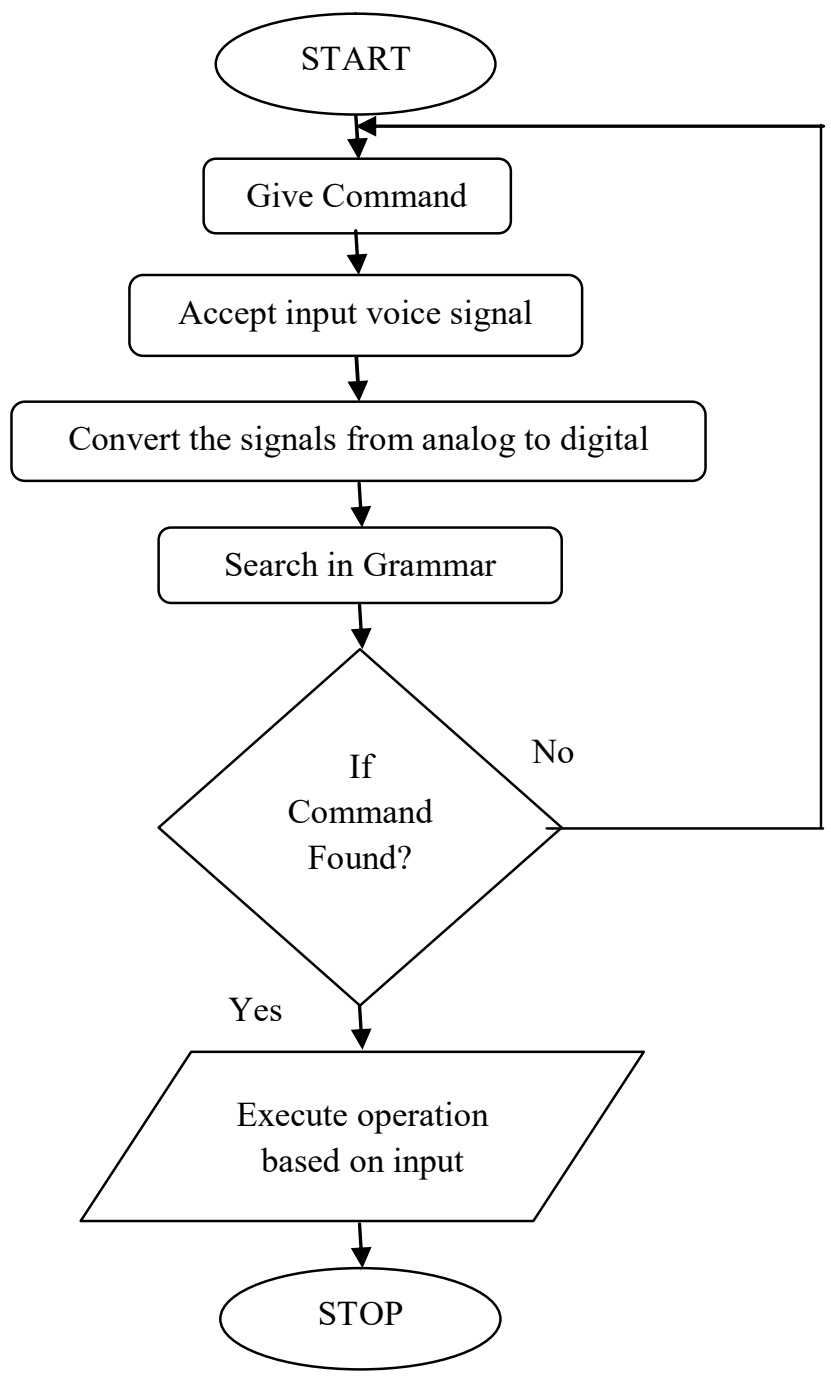

\subsection{Algorithm}

- Start

- Give Instructions (Speak Command).

- Accept the voice input through sound card.

- Convert the voice analog signal into digital signal.

- Search the Grammar to identify whether signal (command) is matching or not.

- If the command not found go to step 2 .

- Execute operations based on the match found from grammar (instructions retrieved from matched

- Value).

- Stop

\section{FUTURE WORK}

Today, when we call most telephone line providers, ISPs, or customer care, we can hear an automated voice recording which instructs you to press buttons to move through some option menus. There are some companies which provide similar system, but instead of you pressing the buttons you just have to speak to get what you need. The system used in these companies is a also type of speech recognition program [4].

Our product is made available for use at home also apart from business uses. 'Control Mouse and Computer System Using Voice Commands'allow user to dictate and computer recognize his spoken words and converts it to text in a word processing application or e-mail document. User can also give some specific commands to perform computer operations, such as opening files and accessing menus, and also mouse related events.

In future we would like to enhance our project in the best possible ways. We plan to add more commands. Enhancement of our dictionary with more words is also our next objective. At present our system works with Windows. However we plan to enable multiplatform support with Linux and Mac Os. We also would like to enable multi language support. Besides, main objective is to improve the accuracy of the system and create full web support.

\section{CONCLUSION}

The 'Control Mouse and Computer System Using Voice Commands' is built with the aim of making easy the operations of a computer system with the help of voice. This system may be useful for a regular user. However since a regular user is accustomed to handling the computer system with the help of a keyboard and a mouse, this system proves to be useful to the physically handicapped individuals who may not be able to operate the computer. Thus the current 'Control Mouse and Computer System Using Voice Commands' developed fulfills this social cause of reaching out to the physically handicapped and ensuring that they too can avail of the functionalities of a computer system. [4]

Our project thus making use of the programming language Visual Basic.NET and Microsoft Speech Application Programming Interface SDK- the speech application programming interface, helps create a completely functional voice driven operating system. We hope this system eases out the complexity of using hardware to operate computer systems and makes it easier to operate them with voice.

\section{REFERENCES}

[1]. Fundamentals of Speech Recognition 1st Edition by Lawrence Rabiner (Author), Biing-Hwang Juang (Author).

[2]. Speech based system. N.K. Vela rum Mohan Ram (Author).

[3]. http://www.phon.ucl.ac.uk/courses/spsci/compmeth/spe ech/recognition.html 
[4]. http://electronics.howstuffworks.com/gadgets/hightech-gadgets/speech-recognition.htm

\section{BIOGRAPHIES}

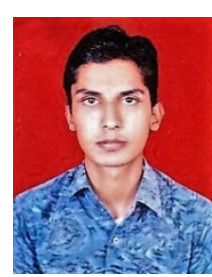

Name: Mr. Mahammad Rafi

Designation: Student

Department: Computer Engineering

Qualifications: B.E.(comp)Pursuing

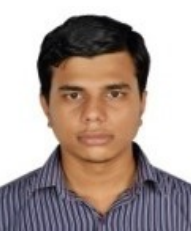

Name: Mr. Khan Sohail

Designation: Student

Department: Computer Engineering

Qualifications: B.E.(comp)Pursuing

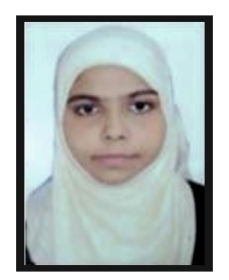

Name: Ms. Shaikh Huda

Designation: Student

Department: Computer Engineering

Qualifications: B.E.(comp)Pursuing

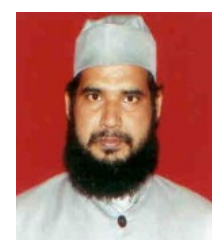

\section{Name: Mr. Md. Lutful Islam}

Designation: Assistant Professor

Department: Computer Engineering

Qualifications: M.C.A, M. Tech (Comp) 